\title{
O uso da metodologia da problematização Arco de Maguerez em uma clínica escola
}

\section{da graduação de Enfermagem}

The use of the Arco de Maguerez Problematization methodology in a Nursing graduation school

clinic

El uso de la metodología de problematización Arco de Maguerez en una clínica escolar de graduación de Enfermería

Recebido: 17/05/2021 | Revisado: 27/05/2021 | Aceito: 02/06/2021 | Publicado: 17/06/2021

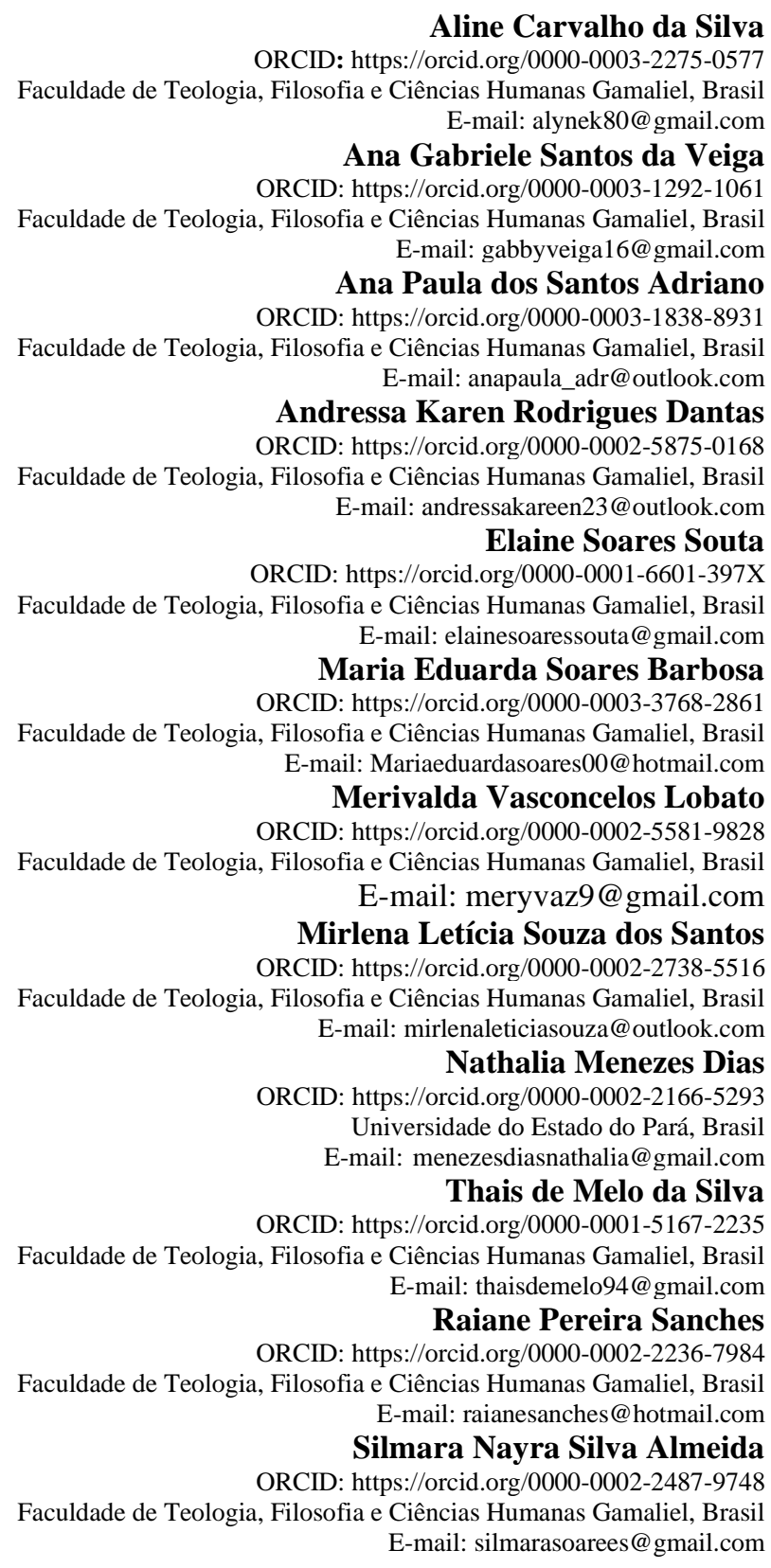

\section{Resumo}

Objetivo: Descrever a experiência de acadêmicos do curso de enfermagem da faculdade Gamaliel diante às dificuldades encontradas durante o estágio curricular, utilizando-se da Metodologia da Problematização (MP) 
associado ao arco de Maguerez. Metodologia: trata-se de um relato de experiência com abordagem reflexiva, caracterizado pela metodologia da problematização (MP) implementada através das cinco etapas do Arco de Maguerez. Resultados e Discussão: a experiência resultou no desenvolvimento de uma ficha objetiva para controle da produção de enfermagem local, a fim de agilizar o processo de triagem e agregar informações necessárias para nortear o plano de cuidado da enfermagem local, além de disponibilizar dados confiáveis para a comunidade acadêmica desenvolver estudos científicos na área da saúde. Conclusão: dado o exposto, entende-se que a produção de enfermagem é um fator essencial na compreensão dos determinantes em saúde, portanto o desenvolvimento dessa tecnologia otimizaria o tempo de triagem tornando mais eficiente, direcionando dados concretos para nortear planos de cuidados eficazes, além de fomentar estudos científicos voltados a área da saúde fortalecendo a tríade ensino, pesquisa e extensão importante para a construção de conhecimento aos acadêmicos.

Palavras-chave: Enfermagem; Arco-Maguerez; Tecnologia.

\begin{abstract}
Objective: To describe the experience of academics in the nursing course at Gamaliel College in view of the difficulties encountered during the curricular internship, using the Problem-solving Methodology (PM) associated with the Maguerez arch. Methodology: this is an experience report with a reflective approach, characterized by the methodology of problematization (MP) implemented through the five stages of the Arco de Maguerez. Results and Discussion: the experience resulted in the development of an objective form to control the local nursing production, in order to streamline the screening process and add information necessary to guide the local nursing care plan, in addition to providing reliable data for the academic community to develop scientific studies in the health area. Conclusion: given the above, it is understood that nursing production is an essential factor in understanding health determinants, so the development of this technology would optimize the screening time making it more efficient, directing concrete data to guide effective care plans, in addition to to promote scientific studies focused on the health area, strengthening the triad of teaching, research and important extension for the construction of knowledge to academics.
\end{abstract}

Keywords: Nursing; Arco-Maguerez; Technology.

\title{
Resumen
}

Describir la experiencia de los académicos del curso de enfermería de la Facultad Gamaliel ante las dificultades encontradas durante la etapa curricular, utilizando la Metodología de Problematización (MP) asociada al arco de Maguerez. Metodología: Se trata de un informe de experiencia con un enfoque reflexivo, caracterizado por la Metodología de Problematización (PM) implementada a través de los cinco pasos del Arco de Maguerez. Resultados y Discusión: la experiencia resultó en el desarrollo de un formulario objetivo para el control de la producción de la enfermería local, con el fin de agilizar el proceso de detección y añadir la información necesaria para orientar el plan de atención de la enfermería local, además de proporcionar datos fiables para la comunidad académica para desarrollar estudios científicos en la salud. Conclusión: Teniendo en cuenta lo anterior, se entiende que la producción de enfermería es un factor esencial en la comprensión de los determinantes de la salud, por lo que el desarrollo de esta tecnología optimizaría el tiempo de triaje, haciéndolo más eficiente, dirigiendo datos concretos para orientar los planes de atención eficaces, además de fomentar los estudios científicos centrados en el área de la salud, el fortalecimiento de la tríada de la enseñanza, la investigación y la extensión, que es importante para la construcción del conocimiento para los estudiantes.

Palabras clave: Enfermería; Arco-Maguerez; Tecnología.

\section{Introdução}

Para Amaral et al (2012) as clinicas escolas são consideradas instituições privadas oriundas de universidades, onde sua atuação vem colaborar na assistência à saúde na sociedade, ao ofertar serviços especializados através de equipes multiprofissionais, e que também atuam no desenvolvimento profissional de acadêmicos, uma vez que exercem suas atividades diretamente em sua área de estudo.

Estes locais são conceituados por serem responsáveis por grande parte da expansão acerca dos conhecimentos científicos na enfermagem, sendo estrategicamente elaborados para que os estudantes possam absorver e utilizar seus conhecimentos, aplicando na prática e ampliando assim sua visão crítica sobre situações problemáticas que instantaneamente podem atrasar ou prejudicar a prestação dos serviços, bem como, a promoção da saúde nas comunidades assistidas (Martins et al., 2012).

Nesse sentido, Flores e Pedroso (2014) afirmam que a importância da clínica escola está voltada ao ensino, visto que possibilita ao aluno adquirir em meio à prática em campo ao articular com pesquisa, extensão e ensino, as habilidades clinicas 
necessárias para sua formação, bem como a sua interação em uma equipe multiprofissional, desenvolvendo questões éticas frente ao cuidado com o paciente.

Dessa forma, é comum utilizações de metodologias que possibilitem desafios enriquecedores para o conhecimento, destaca-se que a Metodologia da Problematização (MP) busca preparar os estudantes para tornarem-se aptos a observar a realidade e conseguir conduzir uma possível resolução dos problemas, contribuindo para as melhorias em seu campo de atuação (Dalla, Moura \& Bergamaschi, 2015).

Mediante as fragilidades dos acadêmicos diante da inexperiência em campo, este método surge como uma estratégia de ensino-aprendizagem que produz sedimentação teórico-prática por meio da construção do conhecimento, além de influenciar nas habilidades interpessoais com a equipe de trabalho e com os usuários do serviço de saúde, que ocorre através da compreensão da realidade das pessoas de forma holística e humanizada (Macedo et al., 2018).

A sua concepção teórico-crítica visa preparar o acadêmico para a tomada de decisão quando se obtêm a consciência de seu mundo, contudo, para isso é utilizado o Arco de Maguerez que é considerado uma estratégia de ensino que precede o desenvolvimento da problematização, sendo descrita através de cinco etapas (Prado, Velho, Espíndola, Sobrinho, \& Backes, 2012).

Sabendo que durante a observação crítica destas cinco etapas é possível identificar problemas reais relacionados ao desenvolvimento das práticas na enfermagem, diante da grande demanda de pacientes que utilizam os serviços da instituição que são ofertados, sendo atendimentos executados por profissionais com diversificadas especialidades (Castro et al., 2018).

Visto isso, este estudo tem como objetivo relatar a experiência dos acadêmicos no uso da Metodologia da Problematização (MP) com o arco de Maguerez frente as dificuldades encontradas durante o estágio curricular na estruturação quanto controle de produção da equipe de enfermagem.

\section{Metodologia}

Trata-se de um estudo do tipo relato de experiência com análise reflexiva, caracterizado por meio da MP através do uso do Arco de Maguerez que é demonstrado segundo Bordenave e Pereira (2004) através das cinco etapas: sendo a primeira Observação da realidade; a segunda Identificação dos Pontos chave; Teorização na terceira etapa; na quarta etapa a Hipótese de solução e na quinta etapa a aplicação à Realidade.

Para Berbel e Gamboa (2012) este método desenvolvido por Charles Maguerez, sendo o Arco de Maguerez, em adjunto com a MP articulam-se com teoria e prática em variados níveis de conhecimento e aplicabilidade. Diante disso, buscou-se utilizar o devido método uma vez que este proporciona uma das melhores formas de intervenção à realidade.

Iniciou-se o trabalho partir da observação da docente e dos acadêmicos do curso de Bacharel em Enfermagem da faculdade Gamaliel frente a necessidade de uma devida organização dos serviços assistenciais realizados pela equipe, após a aplicação da problematização na unidade.

O processo de ensino-aprendizado aconteceu durante um período de cinco dias, no turno da manhã, junto a profissional responsável pelo grupo de acadêmicos, que atendeu as necessidades propostas aos estudantes, bem como, o manejo da triagem e a elaboração estratégica de conteúdo para a educação em saúde fornecida aos pacientes que se encontravam presentes.

Com isso, a proposta metodológica relatada buscou definir as dificuldades observadas durante o processo de aprendizado vivenciado, contribuindo para a reflexão e construção do conhecimento cientifico sobre a temática, uma vez que enfatiza todos os pontos relacionadas a atuação preconizada pela equipe de enfermagem dentro de uma clínica escola. 


\section{Resultados e Discussão}

Ao conhecer as etapas do método, evidenciou-se conforme abordado pelos autores abaixo, os resultados são apresentados nas cinco etapas do arco de Maguerez descritas a seguir:

PRIMEIRA ETAPA: Para dar início a primeira etapa do arco de Charles de Maguerez foi realizado uma observação da realidade para que pudéssemos identificar uma possível situação problema, ou seja, alguns pontos relevantes durante a atividade curricular ocorrida na unidade, a partir de fatores associados a atuação do profissional enfermeiro e o controle de dados.

A partir de uma reflexão prolongada, observou-se que as atividades desenvolvidas ocorreram de forma dinâmica, instigando os estudantes na busca por situações que necessitavam de urgência primordial, levando em questão todos os eventos ocorridos no local, conforme todas as funções de competência da equipe de enfermagem, como também as palestras e educação em saúde que foram realizadas no período.

Entretanto, as atividades realizadas na unidade referem-se apenas ao momento da triagem, onde coleta-se os sinais vitais e o Índice de Massa Corporal (IMC), porém, o acesso dos pacientes a informação sobre à saúde com base no olhar holístico dos profissionais de enfermagem poderiam ser amplamente melhorados.

Dentre algumas das observações feitas, considerou-se como déficit a falta da disposição de uma tecnologia na sala de triagem que pudesse oferecer de modo objetivo uma maneira de conhecer mais sobre o atendimento ao qual o cliente veio buscar na clínica, além de outros dados como, idade, sexo, procedência, etc. Sabe-se que esses dados são de suma importância com relação a produção de enfermagem, além disso são cruciais para a execução da triagem de forma holística, e desse modo a coleta de informações ocorreria de forma direcionada, eficaz e prática.

Dessa forma, avalia-se como principal problema oriundo da observação da realidade a falta de dados relacionados ao perfil de seus pacientes que interfere na atuação da equipe de enfermagem, que caracteriza seus serviços de forma tênue e generalizada.

SEGUNDA ETAPA: Foi realizada uma síntese da situação inicial, para identificar os fatores condicionantes do problema evidenciado. Esclarecendo os seguintes pontos-chave associados a problemática, nos quais evidenciam-se: $1^{\circ}$ (primeiro)o acesso limitado dos profissionais de enfermagem na monitorização dos casos que chegam a unidade, bem como, aos recursos utilizados diante da elaboração de protocolos e/ou materiais do ambiente; $2^{\circ}$ (segundo) a grande demanda de pacientes na unidade, considera-se um importante viés quanto ao controle específico de dados que são fornecidos, visto que toda produção obtida é direcionada para outro órgão de saúde, refletindo na baixa oferta de informações na clínica.

Ao evidenciar isso, considera-se que o momento da triagem é qualificado como o início do processo de avaliação e abordagem clínica, onde necessitasse de uma estruturação de controle do tempo e sistemas com protocolos de informações devidamente adequados ao local (Moreira, 2010).

No intuito de analisar a atuação da equipe quanto ao seu tempo de prestação de serviços, surgiu a necessidade de acompanhar a quantidade de atendimentos realizados, visto que o funcionamento da clínica dispõe de uma grande quantidade de pacientes, devido as especialidades ofertadas na unidade serem escassas no município e regiões vizinhas. Mediante isto, foi possível coletar o quantitativo de atendimentos realizados no mês de agosto, conforme demonstrado no Gráfico 1: 


\section{Gráfico 1.}

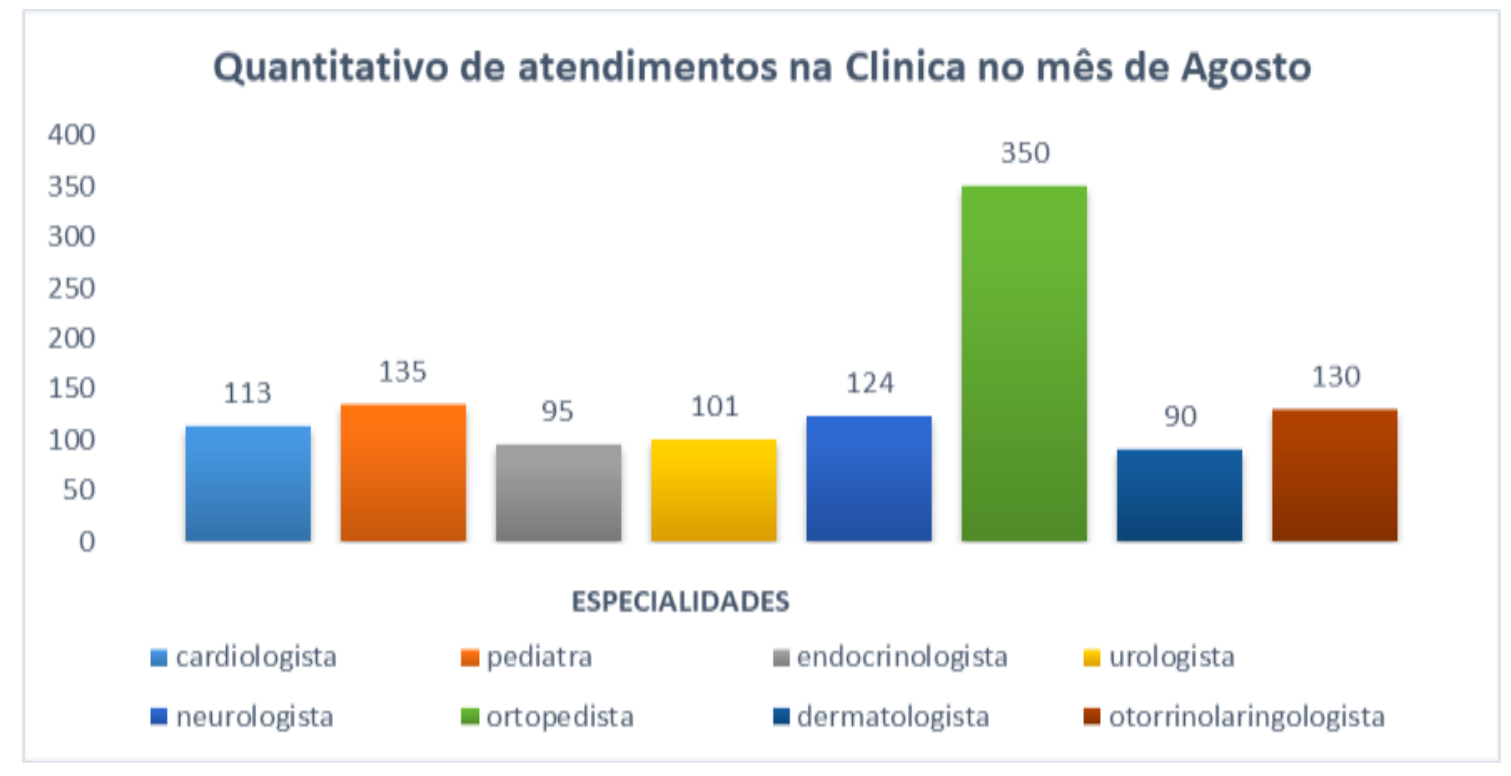

Fonte: Acervo dos autores (2020).

De acordo com o gráfico acima, foi constatado um total de 1.138 atendimentos caracterizando todas as especialidades. De forma individualizada, para o cardiologista houve 113 pacientes, ao pediatra 135, ao endocrinologista 95, ao urologista 101, ao neurologista 124 , ao ortopedista 350 , ao dermatologista 90 , e ao otorrinolaringologista 130 , determinando todas as consultas realizadas.

Com isso, entendeu-se que o local compõe um diversificado trabalho multiprofissional, onde os especialistas atuam, prevenindo, tratando e reabilitando inúmeros casos de pacientes da região, possuindo assim, uma grande demanda de atendimentos mensais, que está diretamente relacionado ao acolhimento da enfermagem, pois, precede com a execução dos primeiros cuidados que são estabelecidos em prol do paciente.

TERCEIRA ETAPA: Nesta etapa do arco de Maguerez os sujeitos da pesquisa refletem sobre o porquê dos problemas levantados anteriormente com bases teóricas, no entanto, é através de uma boa teorização que se encontra o fundamento cientifico para resolver a problemática em questão, no qual vem causando o déficit na qualidade dos serviços ou situações demonstradas (Prado et al., 2012).

Visto isso, foi realizada uma busca de informação referentes aos pontos-chave para que pudéssemos ter o devido embasamento cientifico na literatura proveniente de artigos científicos publicados em revistas ou plataformas acadêmicas. No qual levantou-se as seguintes reflexões norteadoras.

A pratica da enfermagem é preconizada com base nas ações de atendimento realizadas desde o momento de entrada até a saída do paciente da unidade, sendo estas práticas executadas de acordo com situações específicas que cada cliente apresentar, logo, exige-se um raciocínio clínico e habilidades para ofertar as intervenções cabíveis e conhecimento especializado diante do tipo de recorrência (Oliveira, 2020).

Sabendo que o profissional de enfermagem que esteja atuando na triagem deve possuir capacitação, para as situações comuns ao serviço, como a preservação e valorização das necessidades biológicas, sociais e psicológicas dos pacientes, por meio de senso crítico diante de cada caso, com isso, exercendo além do conhecimento cientifico adquirido, o papel de liderança que lhe é atribuído (Coutinho, Cicilio \& Mota, 2012).

Nesse contexto, é primordial que se tenha domínio sobre suas atribuições, sendo algumas destacadas, como avaliar recursos utilizados na unidade, reduzir tempo de espera elaborando estratégias para atendimento ágil e eficiente, acompanhar 
em casos de necessidades, orientar os pacientes quanto aos protocolos e procedimentos da unidade e conhecer os aspectos culturais e socioeconômico de forma individualizada (Lima \& Paula, 2017).

No entanto, para que exerçam sua atividade com excelência faz-se fundamental conhecer o funcionamento da clínica, quanto aos serviços prestados em todas as etapas do cuidado, e então possuir um método eficaz para coletar os dados sobre o histórico do paciente, conseguindo assim avaliar fatores agravantes que podem ser resolvidos, uma vez que conhecer seu público torna o serviço centralizado e objetivo reduzindo-lhe os sofrimentos (Acosta, Duro \& Lima, 2012).

Segundo Jorge et al., (2012) um fator agravante nos serviços hospitalares é a superlotação, isso acontece quando há falta de recursos humanos, serviços especializados e materiais nos outros estabelecimentos de atenção à saúde, como hospitais e Unidade de Pronto Atendimento (UPA), que consequentemente faz com que as pessoas recorram a unidades com serviços especializados em busca de um atendimento qualificado.

De acordo com Venturini, Decésaro e Marcon (2007) para cada necessidade que o paciente apresentar, cabe a equipe desenvolver medidas estruturadas para introduzir no atendimento, na intenção de reduzir os impactos provocados pelo acometimento da doença ou trauma.

Para o devido planejamento da assistência à saúde surgiram muitas inovações que fomentam a criação de instrumentos para auxiliar no processo de cuidado, seja em meios lineares ou complexos, a busca por estas novas tecnologias quando associadas ao contexto da estrutura física de uma unidade e aos processos de trabalho multidisciplinar existentes, pode ser um grande facilitador do acesso a saúde (Salvador, Oliveira, Costa, Santos, \& Tourinho, 2011).

Mediante os autores citados, viu-se muitos fatores que refletem o atendimento do enfermeiro, então atentou-se para a busca de um processo metodológico que pudesse acompanhar os clientes assistidos na clínica por meio do âmbito técnicocientifico, de forma planejada e sistemática.

Sabendo que a criação de tecnologias facilita a prática profissional, ressalta-se também que a ausência dessas produções compromete a estruturação do atendimento e o manejo do cuidado, além de afetar a sensibilização da equipe para as situações que necessitam do olhar holístico de um enfermeiro (Cardoso, 2020).

QUARTA ETAPA: Inicia-se nesta etapa a elaboração da hipótese de solução para a problemática, a partir do levantamento e reflexão teórica-cientifica realizada anteriormente, com a intenção de planejar uma solução plausível através de métodos criativos, levando em consideração a relação entre teoria e pratica vivenciada (Vieira \& Panúncio, 2015).

Após uma reunião realizada entre os acadêmicos e a profissional de enfermagem, foi idealizada a criação de uma ficha de produção de enfermagem que atendesse aos critérios mínimos de tempo e objetividade. Uma estrutura que visa atender de acordo com o tempo concedido para a equipe, sem afetar a agilidade dos atendimentos.

Para a objetividade pontuou-se as vertentes mais importantes a serem coletadas, bem como, a especialidade a ser consultada, as comorbidades que o paciente apresenta, fatores socioeconômicos e dados específicos relacionados a cor, gênero e local de moradia, tornando o momento de coleta prático e eficaz, desejando fomentar o acervo de dados relacionado à produção de enfermagem.

QUINTA ETAPA: Neste momento validado como a aplicação à realidade do instrumento idealizado para solucionar a problemática, ocorre o surgimento de novas perspectivas que podem modificar o ambiente com base na hipótese levantada (Santos, Feitosa, Ribeiro, \& Cavalcante, 2018).

A criação deste material classificado como a ficha de produção a ser utilizada durante o atendimento de enfermagem, seja de acadêmicos ou profissionais de enfermagem, deu-se através do programa operacional "Microsoft Word" tubulado em tabela, formato A4, modo retrato. Disponibilizado na unidade por meio de material impresso e digitalizado, durante um período de 2 meses, seguindo as etapas do Arco de Maguerez, conforme demonstrado no Fluxograma 1: 
Fluxograma 1. O Arco da problematização de Charles Maguerez.

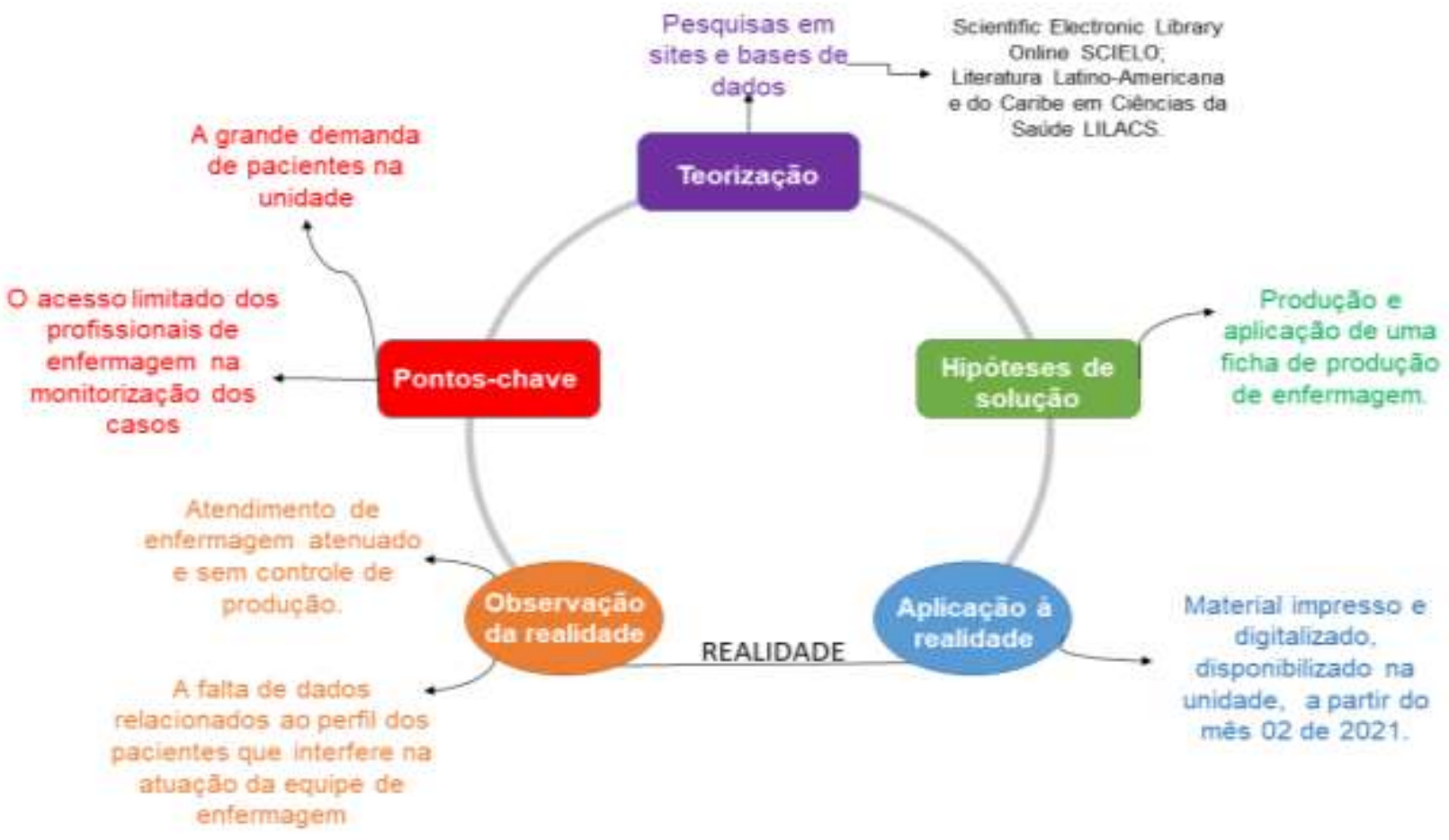

Fonte: Acervo dos autores (2020).

Mediante o fluxograma apresentado, observa-se a sequência das cinco etapas do arco de Maguerez, que propõem como resultado a finalização da ficha de produção para os profissionais e acadêmicos de enfermagem, onde conforme descrito na quinta etapa foi disponibilizada na unidade em questão.

Para facilitar o uso do material, foi sugerido para o enfermeiro da unidade que a ficha fosse anexada na sala de triagem, o que proporcionara aos estudantes mais agilidade no processo de preenchimento para atender todos os clientes.

Uma vez sabendo das atribuições do enfermeiro como membro ativo na unidade, sendo capaz de gerenciar recursos e alinhar o funcionamento da equipe, ao atuar idealizando boas táticas tecnológicas em saúde, torne-se perceptível que tais avanços influência no desenvolvimento de diversos setores, incluindo o setor de triagem (Maia et al., 2021).

Dessa forma, nesta etapa os estudantes refletiram sobre os conhecimentos prévios quanto ao uso da tecnologia e a oferta de dados atribuídos a equipe, que irá promover boas condições de estudos e cenário para a equipe desenvolver habilidades e senso crítico.

\section{Conclusão}

Diante dos fatos mencionados, sabe-se que a produção de enfermagem é um instrumento essencial para a compreensão de diversos fatores relacionados ao estado de saúde dos clientes da Clínica Escola, com base nisso observou-se a necessidade implícita da criação de uma tecnologia de auxílio, desse modo o enfermeiro responsável pela sala de triagem conseguiria observar os clientes de modo holístico, e através dos dados coletados para a produção conseguirá ser mais preciso quanto a sua avaliação, visto que se um cliente vai a consulta com o cardiologista é imprescindível que se faça a avaliação antropométrica, pois a avaliação da circunferência abdominal é um dos indicadores de alerta quanto a problemas cardiovasculares. 
Diante disso, é de fato necessário compreender a demanda local, para que os serviços oferecidos dentro da Clínica possam abranger o cliente como um todo, coletando todas as informações necessárias a fim de influenciar positivamente nas consultas dos especialistas. Desse modo, ressalta-se que através da tecnologia da ficha de produção o enfermeiro conseguirá desempenhar sua função com mais eficiência, visando sempre o bem-estar do cliente, comandando o ato da triagem que pode ser considerado um trabalho simples por muitos, mas que é também uma das etapas mais importantes do cuidado.

Portanto, compreende-se que a utilização de uma tecnologia assistencial também fornece inúmeros dados que podem servir como um mediador para as melhorias dos serviços de saúde, uma vez que através dos dados obtidos podem ser realizadas pesquisas voltadas para o entendimento e resolução dos problemas apresentados dentro da unidade, onde posteriormente, podem desempenhar um papel de referência na literatura para outras instituições.

\section{Referências}

Acosta, A. M., Duro, C. L., M., \& Lima, M. A. D. S. (2012). Atividades do enfermeiro nos sistemas de triagem/classificação de risco nos serviços de urgência: revisão integrativa. Revista Gaúcha de Enfermagem, 33(4), 181-190.

Amaral, A. E. V., Luca, L., Rodrigues, T. C., Leite, C. A., Lopes, F. L., \& Silva, M. A. (2012). Serviços de psicologia em clínicas-escola: revisão de literatura. Boletim de Psicologia, 62(136), 37-52.

Berbel, N. A. N., \& Sánchez Gamboa, S. A. (2011). A metodologia da problematização com o Arco de Maguerez: uma perspectiva teórica e epistemológica. Filosofia E Educação, 3(2), 264-287.

Bordenave, J. D., \& Pereira, A. M. P. (2004). Estratégias de ensino-aprendizagem. Vozes.

Castro, C. S., Pantoja, E. M. S., Lupselo, S. R., Maritê, I. A., Hoffmann, A. C. O. S., \& Bastiani, J. (2018). Fatores que influenciam no cuidado seguro de enfermagem ao paciente. Revista Eletrônica Estácio Saúde, 7, 32-39, 2017.

Cardoso, S. V. (2020). Tecnologia de cuidado de Enfermagem para redução do estresse em pacientes internados em Unidade de Terapia Intensiva.

Coutinho, A. A. P., Cecílio, L. C. O., \& Mota, J. A. C. (2012). Classificação de risco em serviços de emergência: uma discussão da literatura sobre o sistema de triagem de Manchester.Revista Medica de Minas Gerais, v. 22, n. 2, p. 188-98.

Dalla, M. D. B., Moura, G. A. G., \& Bergamaschi, M. S. (2015). Metodologias ativas: um relato de experiência de estudantes de graduação em medicina da Universidade Vila Velha na disciplina de Interação Comunitária.Revista Brasileira de Medicina de Família e Comunidade, $10,1-6$.

Flores, E. R. M., \& Pedroso, J. S. (2014). Triagem em clínica escola: estudos das diversas práticas. Psicologia Argumento, 32(78).

Gentil, R. C., Ramos, L. H., \& Whitaker, I. Y. (2008). Capacitação de enfermeiros em atendimento pré-hospitalar. Revista Latino-Americana de Enfermagem, 16 (2), 192-197.

Jorge, V. C., Barreto, M. S., Ferrer, A. L. M, Santos, E. A. Q., Rickli, H. C., \& Marcon, S. S. (2012). Equipe de enfermagem e detecção de indicadores de agravamento em pacientes de pronto-socorro. Escola Anna Nery, 16(4), 767-774.

Lima, S., \& Paula, A. S. (2017). Atuação do enfermeiro na aplicação do Protocolo de Manchester em unidades de urgência e emergência. ANAIS SIMPAC, 8(1).

Macedo, K. D. S., Acosta, B. S., Silva, E. B., Souza, N. S., Beck, C. L. C., \& Silva, K. K. D. (2018). Metodologias ativas de aprendizagem: caminhos possíveis para inovação no ensino em saúde. Escola Anna Nery, 22(3), e20170435.

Maia, A. R. B., Andrade, C. N. D., Nascimento, G. B., Rodrigues, G. T., Fernandes, J. M., Oliveira, K. L., Valinho, P. B., Marinho, A. M., Rocha, R. G., Xavier, M. L., \& Marta, C. B. (2021). Relação teórico-prática da administração em enfermagem vivenciada em uma unidade de saúde: relato de experiência. GlobAcadNurs. 2021;2(1):e77.

Martins, J. C. A., Mazzo, A., Baptista, R. C. N., Coutinho, V. R. D., Godoy, S., Mendes, I. A. C., \& Trevizan, M. A. (2012). A experiência clínica simulada no ensino de enfermagem: retrospectiva histórica. Acta Paulista de Enfermagem, 25(4), 619-625.

Moreira, C. T. P. (2010). Avaliação de uma implementação do Sistema de Triagem de Manchester. Que realidade? Dissertação de Mestrado apresentada à Faculdade de Medicina Universidade de Porto, Porto, Portugal.

Oliveira, J. C. N. D. (2020). Percepção do enfermeiro acerca das dificuldades e de sua capacitação profissional para atuar no serviço de atendimento móvel de urgência-SAMU.

Prado, M. L., Velho, M.B., Espíndola, D. S., Sobrinho, S. H., \&Backes, V. M. S. (2012). Arco de Charles Maguerez: refletindo estratégias de metodologia ativa na formação de profissionais de saúde. Escola Anna Nery, 16(1), 172-177.

Salvador, P. T. C. O., Oliveira, R. K. M., Costa, T. D. C., Santos, V. E. P., \& Tourinho, F. S. V. (2011). Tecnologia e inovação para o cuidado em enfermagem. Revista Enfermagem UERJ, 20(1), 111-117. 
Research, Society and Development, v. 10, n. 7, e15410716194, 2021

(CC BY 4.0) | ISSN 2525-3409 | DOI: htttp://dx.doi.org/10.33448/rsd-v10i7.16194

Santos, K. C. B., Feitosa, A. H. C., Ribeiro, G. S. C., \& Cavalcante, T. B. (2018). Metodologia da problematização com Arco de Maguerez no centro cirúrgico oftalmológico de um hospital universitário. ReonFacema.

Venturini, D. A., Decésaro, M. N., \& Marcon, S. S. (2007). Alterações e expectativas vivenciadas pelos indivíduos com lesão raquimedular e suas famílias. Revista da Escola de Enfermagem da USP, 41(4), 589-596.

Vieira, M. N. C. M., \& Panúncio, P. M. P. (2015). A Metodologia da Problematização (MP) como estratégia de integração ensino-serviço em cursos de graduação na área da saúde. Medicina (Ribeirão Preto), 48(3), 241-248. 